\section{Revista Chilena \\ de Pediatría}

www.revistachilenadepediatria.cl

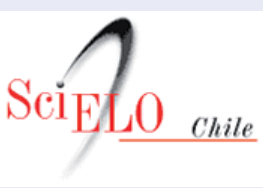

www.scielo.cl

\title{
Ley de Etiquetado y Publicidad de Alimentos: Chile innovando en nutrición pública una vez más
}

\author{
Law of Food Labelling and Advertising: \\ Chile innovating in public nutrition once again
}

\author{
Lorena Rodríguez Osiac ${ }^{\mathrm{a}}$, Tito Pizarro Quevedo ${ }^{\mathrm{b}}$
}
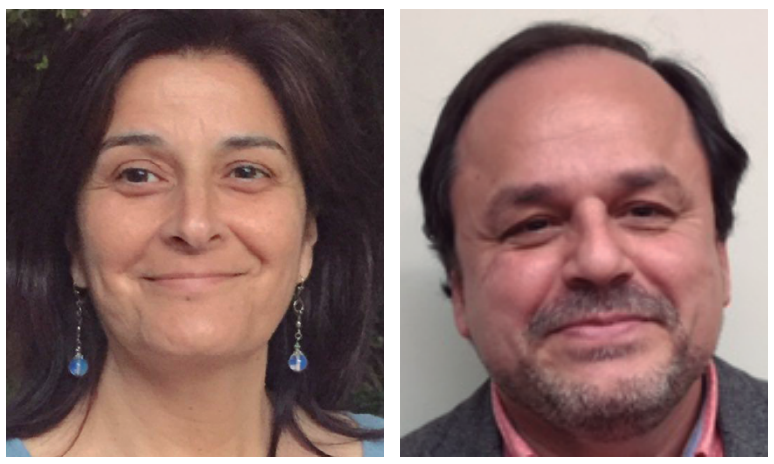

aEscuela de Salud Pública, Universidad de Chile

bDepartamento de Salud Pública, Universidad de Santiago

Como es de público conocimiento, gracias a innovadoras medidas de salud pública, especialmente en el ámbito de la pediatría, Chile fue de los primeros países en el mundo en superar la desnutrición y junto con ello disminuir la mortalidad infantil. De esa exitosa historia somos como sociedad herederos. Sin embargo no vimos venir los cambios en el estilo de vida y en especial en la dieta que llevaron a un aumento veloz en la prevalencia de malnutrición por exceso y de enfermedades vinculadas como diabetes, hipertensión, dislipidemias, cardiovasculares y distintos tipos de cáncer. Hoy tenemos lamentablemente cifras muy altas y en ascenso de estas condiciones, a pesar de los esfuerzos realizados en materia de políticas públicas.

La evidencia científica muestra que el control y prevención del exceso de peso y la promoción de una alimentación saludable, es complejo y multifactorial. Es necesario un enfoque integral desde distintos sectores, así como lo propone la Organización Mundial de la Salud a través del concepto "Salud en Todas las Políticas", y teniendo en consideración los determinantes sociales y comerciales de la salud.

Las estrategias más recomendadas para combatir la obesidad son aquellas que incluyen tanto el abordaje estructural (legislativo, regulatorio, fiscal) de los entornos alimentarios, como el abordaje individual del problema (consejería, guías y recomendaciones alimentarias). No basta con educar a la población en su autocuidado, ni con el tratamiento de la obesidad y sus complicaciones, sino que se debe asegurar que el ambiente político, económico, social y cultural en el que transcurre la vida de las personas, y que incide en su comportamiento, favorezca y permita las decisiones más saludables, por ejemplo con información clara y sencilla de la composición de los alimentos (etiquetado), regulando la publicidad de los alimentos y su venta en espacios escolares, medidas fiscales que modifiquen los precios de los alimentos, aumento de la oferta y acceso a productos saludables, y otras medidas de esta misma índole.

En este contexto, el Estado de Chile se ha hecho cargo de esta situación y desde hace más de 15 años que viene implementando acciones tanto en el ámbito individual a través del control de salud, del control de enfermos crónicos, de la consulta nutricional y de programas como el "Vida Sana" que atiende en todo el país a niños, niñas, jóvenes y adultos con malnutrición por exceso; como en el ámbito comunitario a través de programas de promoción de la salud como el programa Vida Chile, la estrategia EGO Chile, el Siste- 
Figura 1. Sello para etiquetado frontal de advertencia en los alimentos envasados.

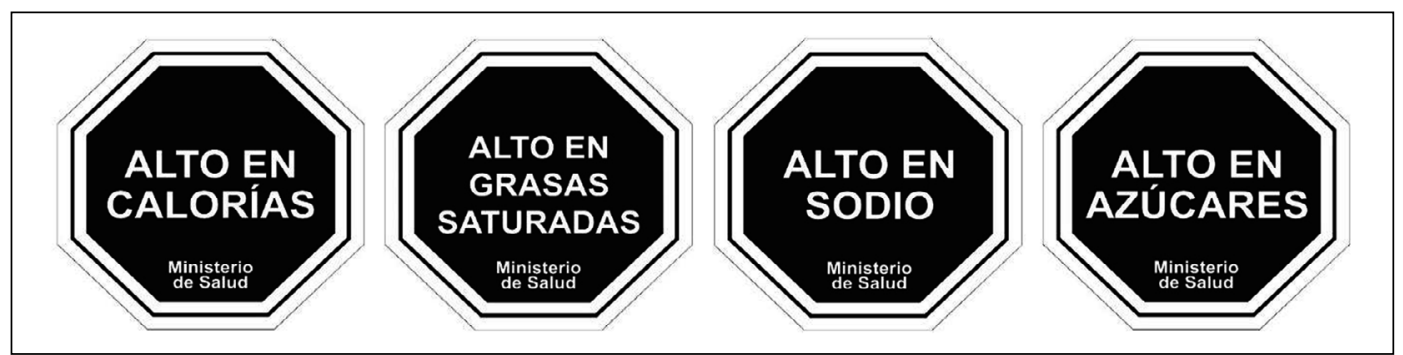

ma de Protección Social ChileCreceContigo y las más recientes Estrategia Municipios, Comunas y Comunidades Saludables y el Sistema Elige Vivir Sano; y también en el ámbito estructural a través de los Programas Alimentarios Estatales (PNAC, PACAM, PAE); de la regulación del contenido de grasas trans en los alimentos; de nuevas leyes como la Ley 20.606 de Etiquetado y Publicidad de Alimentos, la Ley 20.869 de Publicidad (complementaria con la anterior); y de medidas fiscales como los impuestos a las bebidas azucaradas. Entre lo más destacado figura la recién lanzada Política Nacional de Alimentación y Nutrición, que entrega el marco de referencia para el desarrollo de regulaciones, estrategias, planes, programas y proyectos en materia de alimentación y nutrición, recogiendo con fuerza el concepto del derecho a una alimentación que satisfaga las necesidades, pero que al mismo tiempo sea de calidad, inocua y culturalmente aceptada.

La Ley 20.606 sobre la Composición Nutricional de los Alimentos y su Publicidad, también conocida como Ley de Alimentos o Ley de Etiquetado, es una de las medidas de salud pública más innovadoras y osadas de los últimos años. Esta Ley nace de una propuesta Parlamentaria apoyada por el Ejecutivo, con el objetivo principal de proteger la salud de niños y niñas modificando los entornos alimentarios que los rodean, así como de favorecer la selección informada de alimentos y disminuir el consumo de alimentos con exceso

Tabla 1. Límites de nutrientes críticos en alimentos

\begin{tabular}{lccc}
\hline Alimentos sólidos & Junio 2016 & Junio 2018 & Junio 2019 \\
\hline Energía kcal/100 g & 350 & 300 & 275 \\
Sodio mg/100 g & 800 & 500 & 400 \\
Azúcares totales g/100 g & 22,5 & 15 & 10 \\
Grasas saturadas g/100 g & 6 & 5 & 4 \\
\hline Alimentos líquidos & Junio 2016 & Junio 2018 & Junio 2019 \\
\hline Energía kcal/100 ml & 100 & 80 & 70 \\
Sodio mg/100 ml & 100 & 100 & 100 \\
Azúcares totales g/100 ml & 6 & 5 & 5 \\
Grasas saturadas g/100 ml & 3 & 3 & 3 \\
\hline
\end{tabular}

de energía y nutrientes críticos (sodio, azúcares, grasas saturadas), con el objetivo a largo plazo de mejorar la condición nutricional de nuestra población.

La Ley 20.606 incluye 5 áreas principales: 1) un nuevo etiquetado frontal de advertencia en alimentos; 2) restricciones a la publicidad de alimentos especialmente aquella dirigida a menores de 14 años; 3) restricciones a la venta de alimentos en establecimientos educacionales de prebásica, básica y media; 4) incorporación de un mensaje que promueva hábitos de vida saludable en la publicidad de algunos alimentos ;y 5) incorporación en todos los niveles educativos, de actividades didácticas que contribuyan a desarrollar hábitos alimentación saludable y de vida activa. De los 4 primeros ejes se hizo cargo el Ministerio de Salud y del 5to el Ministerio de Educación.

Para implementar la Ley 20.606 se redactó un Reglamento que se incorporó al Reglamento Sanitario de los Alimentos (RSA DS 977/96) como parte de la regulación que ya existe en nuestro país hace más de 20 años. El trabajo técnico del Ministerio de Salud incluyó la definición de límites para energía, sodio, azúcares y grasas saturadas; el ámbito de aplicación de la nueva regulación (alimentos e ingredientes afectos); y la definición de publicidad dirigida a menores de 14 años. Para esto se conformaron grupos de trabajo expertos liderados por profesionales del Ministerio de Salud, que realizaron las propuestas que luego fueron discutidas ampliamente y puestas en consulta pública nacional e internacional. Paralelamente se licitaron estudios con consumidores para establecer un sello de advertencia claro y fácil de entender. La propuesta final incluyó el formato de octágono negro (figura 1) con letras blancas con el mensaje "alto en calorías, sodio, azúcares, o grasas saturadas" según corresponda, y la frase "Ministerio de Salud". Además incluyó límites graduales según la tabla 1, y definió que los alimentos afectos serían sólo aquellos adicionados de azúcares, grasas saturadas o sodio, quedando excluidos los alimentos naturales sin adición de estos nutrientes (artículo 120 bis RSA). La definición de publicidad dirigida a menores de 14 años quedó establecida en el artículo 110 bis del RSA en razón al contenido de la publicidad y al porcentaje de audiencia infantil de los programas que la emiten. 
Las evaluaciones de la Ley y su implementación, por parte de distintos grupos académicos, demuestran que la población apoya ampliamente y comprende fácilmente las medidas implementadas y que un porcentaje relevante toma en consideración la presencia de sellos de advertencia en los alimentos para decidir su compra, prefiriendo aquellos con menos sellos o sin sellos. Por otra parte el programa de vigilancia y fiscalización de alimentos llevado a cabo a lo largo de todo el país muestra un porcentaje muy alto de cumplimiento por parte de la industria de alimentos y de los establecimientos que expenden alimentos, así como de los establecimientos escolares. Por último observamos, y la industria así lo refiere también que existe un importante número de alimentos que han reformulado su composición en atención a quedar bajo los límites establecidos. Queda como gran pendiente la evaluación de impacto de esta política, en términos de consumo de alimentos y de prevalencia de obesidad, pero no es esperable que estos cambios sean rápidos, hay que esperar la modificación poblacional de estas variables, lo que evidentemente es un proceso lento que probablemente se detectará primero en los niños y niñas y luego en esta misma generación cuando sea adulta.

Los pediatras tenemos un rol relevante por la gran cantidad de oportunidades de encuentro y educación de nuestros pacientes en el control de salud y en las consultas de morbilidad. Los mensajes que podemos transmitir es elige alimentos con menos sellos y si no lo tienen, mejor; preferir alimentos frescos y naturales y las preparaciones culinarias caseras, compra en ferias libres cada vez que puedas. Especialmente relevante son estas recomendaciones para la alimentación diaria de niños y niñas y para las colaciones escolares. Las Guías Alimentarias Poblacionales y las Guías Alimentarias por grupo etario, del Ministerio de Salud, contienen recomendaciones generales y específicas para los distintos grupos poblacionales. Los 11 mensajes de las guías alimentarias son: 1) Para tener un peso saludable, come sano y realiza actividad física diariamente; 2) Pasa menos tiempo frente al computador o la tele y camina a paso rápido, mínimo 30 minutos al día; 3) Come alimentos con poca sal y saca el salero de la mesa; 4) Si quieres tener un peso saludable, evita el azúcar, dulces, bebidas y jugos azucarados; 5) Cuida tu corazón evitando las frituras y alimentos con grasas como cecinas y mayonesa; 6) Come 5 veces verduras y frutas frescas de distintos colores, cada día; 7) Para fortalecer tus huesos, consume 3 veces al día lácteos bajos en grasa y azúcar; 8) Para mantener sano tu corazón, come pescado al horno o a la plancha, 2 veces por semana; 9) Consume legumbres al menos dos veces por semana, sin mezclarlas con cecinas; 10) Para mantenerte hidratado, toma 6 a 8 vasos de agua al día; y 11) Lee y compara las etiquetas de los alimentos y prefiere los que tengan menos grasas, azúcar y sal (sodio).

Chile se instala como país pionero a nivel mundial en materia de nutrición y alimentación, implementando medidas integrales en distintos ámbitos de acción para modificar los ambientes alimentarios. Tenemos aún muchos desafíos de evaluación de lo realizado y también de diseño e implementación de nuevas medidas para alcanzar un desarrollo más saludable.

\section{Conflicto de intereses:}

Los autores declaran no tener conflicto de intereses.

\section{Referencias}

1. Encuesta Nacional de Salud 2016-2017. http://epi.minsal.cl/encuesta-ens/

2. Sobrepeso y Obesidad OMS 2017. http:// www.who.int/es/news-room/fact-sheets/ detail/obesity-and-overweight

3. Modelo Conceptual de Entornos Alimentarios en Chile 2017. http:// iris.paho.org/xmlui/bitstream/ handle/123456789/34583/v41e1692017. pdf?sequence $=1$

4. Ley 20.606, Reglamento, Evaluación 2017. http://www.minsal.cl/reglamentode-la-ley-de-etiquetado-de-alimentosintroduccion/

5. Política Nacional de Alimentación y Nutrición, Chile 2017. http:// www.bibliotecaminsal.cl/wp/ wp-content/uploads/2018/01/ BVS_POL\%C3\%8DTICA-DEALIMENTACI\%C3\%93N-YNUTRICI\%C3\%93N.pdf
6. NOW AND THEN: The Global Nutrition Transition: The Pandemic of Obesity in Developing Countries. Barry M. Popkin, Linda S. Adair, and Shu Wen NgPublished in final edited form as: Nutr Rev. 2012; 70(1): 3-21. https://www.ncbi. nlm.nih.gov/pmc/articles/PMC3257829/ pdf/nihms336201.pdf

7. Impulsar el enfoque de la Salud en Todas las Políticas en las Américas: ¿Cuál es la función del sector de la salud? Breve guía y recomendaciones para promover la colaboración intersectorial. Washington, DC: OPS, 2015. https://www.paho.org/hq/ dmdocuments/2015/hiap-Brief-Guideand-Recomendations-SPA.pdf

8. Enfoques poblacionales de la prevención de la obesidad infantil. Organización Mundial de la Salud, 2016. ISBN 978924350478 0. http:// apps.who.int/iris/bitstream/hand le/10665/250751/9789243504780-spa.pdf; jsessionid=9C0FF2E8E4CCA2836C246FD
0ADDD0903? sequence $=1$

9. Políticas y programas alimentarios para prevenir el sobrepeso y la obesidad: Lecciones aprendidas. FAO, OPS, OMS, 2018. http://www.fao.org/3/i8156es/ I8156ES.pdf

10. Plan de acción para la prevención de la obesidad en la niñez y la adolescencia 53 Consejo Directivo de la OPS. 66 Sesión del Comité Regional de la OMS. 2014 https://www.paho.org/hq/ dmdocuments/2015/Obesity-Plan-OfAction-Child-Spa-2015.pdf

11. Guías Alimentarias para la población Chilena, Ministerio de Salud, 2013. http://www.minsal.cl/portal/url/item/ dde0bc471a56a001e040010165012224. pdf

12. Guía Alimentación del menor de 2 años y hasta la Adolescencia. http:// www.crececontigo.gob.cl/wp-content/ uploads/2016/01/Guia-alimentacionmenor-de-2.pdf 